\title{
Analysis of temperature and density pedestal gradients in AUG, DIII-D and JET
}

P. A. Schneider ${ }^{1}$, E. Wolfrum ${ }^{1}$, R. J. Groebner ${ }^{2}$, T. H. Osborne ${ }^{2}$, M. N. A. Beurskens $^{3}$, M. G. Dunne ${ }^{4}$, B. Kurzan ${ }^{1}$, T. Pütterich ${ }^{1}$, E. Viezzer ${ }^{1}$, the ASDEX Upgrade Team $^{1}$, the DIII-D Team ${ }^{2}$ and JET EFDA Contributors ${ }^{4}$

${ }^{1}$ Max-Planck-Institut für Plasmaphysik, EURATOM Association, Garching, Germany

${ }^{2}$ General Atomics, PO Box 85608, San Diego, CA 92186-5608, USA

${ }^{3}$ EURATOM /CCFE Fusion Association, Culham Sc. Centre, Abingdon, OX14 3DB, UK

${ }^{4}$ Department of Physics, University College Cork, Association Euratom-DCU, Cork, Ireland

Corresponding Author: philip.schneider@ipp.mpg.de

\begin{abstract}
:
A comparison of the AUG and DIII-D temperature pedestals showed significant differences between electrons and ions. For high collision rates the ions are coupled to the electrons and show very similar pedestal top values and gradients. For lower collision rates both decouple and the ion pedestal becomes less steep. The electron temperature gradient scales linearly with its pedestal top value. This trend is independent of collisionality and plasma shape. The normalized total pressure gradient $\alpha$ shows strong correlations with the plasma shape in a way expected by peeling-ballooning theory. The different behaviours of the electron temperature gradient only and the total pedestal pressure gradient suggests a limit for the electron temperature pedestal different from linear edge MHD stability.
\end{abstract}

\section{Introduction}

The high confinement mode (H-mode) in tokamak plasmas is one of the favoured scenarios for future fusion devices. In order to document and understand the features of the H-mode it is mandatory to analyse a wide range of different plasma parameters. One way to achieve this is to compare tokamak devices with different

\footnotetext{
${ }^{4}$ See the Appendix of F. Romanelli et al., Proc. of the 24th IAEA FEC 2012, San Diego, US
} 
capabilities. In the presented study data is included from ASDEX Upgrade (AUG), DIII-D and JET. Together they cover different machine sizes and thus minor radii $a \in[0.47,0.91] \mathrm{m}$, a wide range of plasma current $I_{\mathrm{p}} \in[0.5,2.7] \mathrm{MA}$, toroidal magnetic field $B_{\mathrm{t}} \in[0.7,2.8] \mathrm{T}$, plasma shape and kinetic plasma properties.

Particularly important in an H-mode plasma is the edge pedestal which is responsible for a significant fraction of the plasma stored energy [1 3]. In the recent years a lot of effort was focused on documenting and understanding the basic properties of the pedestal - its gradients [2,4,5], widths [6-12] and top values [13 15]. This paper focuses on the pedestal characteristics, in particular the gradients, just before the onset of an edge localized mode (ELM) in type-I ELMy H-modes. The analysis in this paper is based on a database obtained with the two-line pedestal characterisation which is described in [12] along with the definitions of the parameters used in this paper. The database in [12] is identical to the one used in this paper. The database contains 69 discharges from AUG, of which 43 have edge ion temperature measurements, 64 discharges from DIII-D and 8 from JET. In Section 2 it is shown that gradients in real space at the outer midplane of $T_{\mathrm{e}}$ and $T_{\mathrm{i}}$ at the outer midplane behave differently. JET was not included in studies of $\nabla T_{\mathrm{i}}$ because no ion temperature data was available for the pedestal. In Section 3 the density and pressure gradients are discussed. In Section 4 the different correlations of the normalized total pressure gradient $\alpha$ are illustrated. In particular, a strong correlation with the plasma shape is observed.

\section{Separation of electrons and ions}

AUG and DIII-D have fairly similar specifications concerning their engineering parameters $a, B_{\mathrm{t}}$ and $I_{\mathrm{p}}$. One significant difference between the two machines is the material of the first wall. The plasma facing components (PFC) of AUG are covered with tungsten (W) while in DIII-D they are made out of carbon (C). The data shown from JET includes only measurements with carbon as PFC. In a recent comparison of AUG data with and without the complete $\mathrm{W}$ wall [16] it was shown that, for similar discharge parameters and no gas puffing, the electron pedestal top temperature $T_{\text {e,ped }}$ and density $n_{\mathrm{e}, \text { ped }}$ vary significantly depending on the wall material. With a $\mathrm{W}$ first wall the density is increased by $10-20 \%$ while at the same time the temperature decreases so a similar electron pressure $p_{e, p e d}$ is obtained. The mechanism causing this effect is not clear, but it results in a significant increase of the electron collisionality $\nu_{\text {e }}$ for plasmas in AUG with full $\mathrm{W}$ wall compared to discharges with a partial carbon wall and otherwise identical discharge parameters. This phenomenon helps to explain the different collisionality regimes observed for $\operatorname{AUG}\left(\nu_{\mathrm{e} \star} \in[0.3,3.8]\right)$ and DIII-D $\left(\nu_{\mathrm{e} \star} \in[0.02,0.8]\right)$. Before the upgrade to a full $\mathrm{W}$ wall in AUG, it was possible to reach collisionalities as low as observed in DIIID. However, these data cannot be included in the following analysis because the 

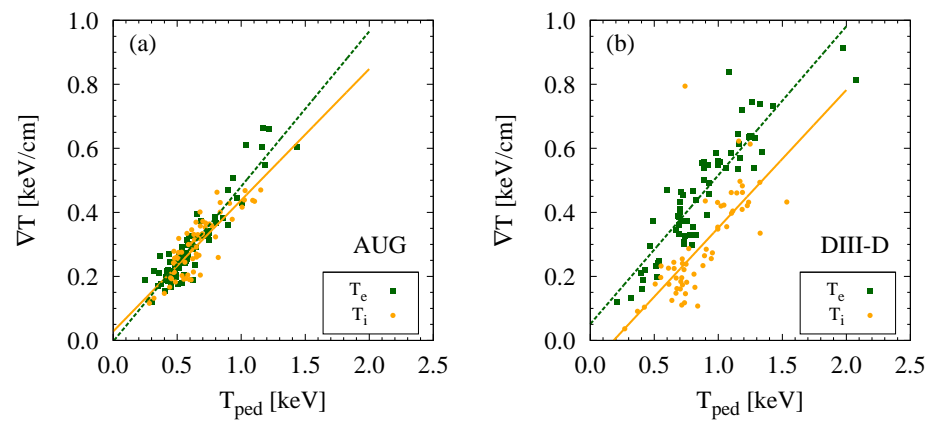

FIG. 1: Pedestal gradient versus pedestal top temperature for electrons (green,square) and ions (orange,circle) with the discharges from AUG (a) and DIII-D (b). The lines are individual linear regressions to the data of the electrons (green, dashed) and ions (orange,solid). The data shown is the same as in Fig. 2, where the separation is done by parameter and not by machine. Fig. 圆also illustrates the uncertainties of the measurement.

high resolution edge charge exchange recombination CER diagnostic [17, which is capable to measure ion temperature gradients in the pedestal, was only installed after the wall upgrade. One has to keep in mind that the CER diagnostic measures the temperature of impurity ions. Fast energy transfer rates between impurity and main ions suggest both are coupled, measurements of the core plasma confirm this assumption [18, but there is no experimental confirmation in the pedestal region.

Temperature gradients of electrons and ions show different behaviour for AUG and DIII-D. In Fig. 1 the pedestal gradient of $T_{\mathrm{e}}$ and $T_{\mathrm{i}}$ is plotted against the temperature at the electron pedestal top. The gradients shown here represent the mean slope in the steep pedestal region in real space at the outer midplane. In the case of AUG (a) electron and ion temperature show a comparable relation between pedestal top value and gradient. For DIII-D (b) the ion temperature gradient is significantly lower than $\nabla T_{\mathrm{e}}$ for comparable temperatures. This is also illustrated by linear regressions which show that electrons (green, dashed) and ions (orange, solid) show the same trend within their uncertainties for AUG, while for DIII-D they have a significant offset from each other. Low collisionality seems to be necessary for the separation of electron and ion temperature. This can be understood when comparing the heat exchange time $\tau_{\mathrm{ei}}$ between electron and ion channel due to collisions with the energy confinement time $\tau_{\mathrm{E}}$. For the given data set the ratio $\tau_{\mathrm{ei}} / \tau_{\mathrm{E}}$ is below 0.1 for $\nu_{\mathrm{e} \star}>0.6$ meaning the heat transfer is fast enough to balance both electron and ion channels before the energy is lost due to heat transport and other mechanisms. For lower collisionalities the heat transfer slows down and becomes $\tau_{\mathrm{ei}} / \tau_{\mathrm{E}} \sim 0.5$ for $\nu_{\mathrm{e} \star} \sim 0.1$. It increases further for 
lower collisionality. The reason why in the presented data set the ion temperature gradient at DIII-D is reduced rather than increased compared to AUG might also be connected to the collision rates in the plasma. However, the presented data does not allow to give a final answer to this question. For more robust conclusions one would need more low collisionality data, in particular, from machines with different size. This would help to separate a real $\nu_{\star}$ dependence from a dependence on temperature and density.

The observations suggest a change in the balance of electron and ion heat channels. This could also be explained with higher collision rates at AUG, where the ion temperature is linked with the electron temperature. The profiles of $T_{\mathrm{e}}$ and $T_{\mathrm{i}}$ can show similar pedestal gradients and pedestal top values. For lower collision rates, as is the case for DIII-D, $\nabla T_{\mathrm{i}}$ can significantly differ from $\nabla T_{\mathrm{e}}$. This implies that the heat transfer between electron and ion channels is not fast enough to guarantee their equilibration and that the electron and ion gradients are set by different physical mechanisms.

Further observations suggest that the ion temperature can be set by the electrons and that the electron temperature is fairly independent of the ions. Fig. 2 (a) shows that the electron temperature gradient scales linearly with $T_{\mathrm{e}, \mathrm{ped}}$. The linear regression to data from AUG, DIII-D and as indicated by the solid line gives $\nabla T_{\mathrm{e}}=0.50 T_{\mathrm{e}, \mathrm{ped}}+0.0015$ and agrees within the uncertainties with the individual of regression AUG and DIII-D data as shown in Fig. 1. This relation is observed independently of variations in machine size, plasma shape, heating power, electron density and ion temperature. Since there is no density dependence at constant $T_{\text {e,ped }}$ there is also no direct collisionality dependence, only an indirect correlation due to the $T_{\mathrm{e}}$ dependence in $\nu_{\mathrm{e} \star}$. This is consistent with observations of the $T_{\mathrm{e}}$ and $n_{\mathrm{e}}$ pedestal width where no significant dependence on collisionality was reported [6, 7, 9 12. The ion temperature gradient shown in Fig. 2 (b) exhibits a much larger scatter for one pedestal top value than the electron temperature. This scatter does not appear for AUG discharges with high collision rates, Fig. 1 (a), and therefore, suggests a stronger coupling between electron and ion heat channels in this case.

\section{Electron density and pressure gradients}

The gradient in the pedestal of the electron density also shows a correlation with the pedestal top value which is consistent for the three machines considered. However, as is illustrated in Fig. 3 (a) the distribution of the measurements is much broader than it was for the temperature. For AUG and JET the external gas puff can influence the correlation of gradient and pedestal top. Fig. 3 (b) shows the AUG and JET data from (a) with the gas puff as color code. With larger gas puff higher pedestal top values are obtained while the gradient remains unchanged. An 

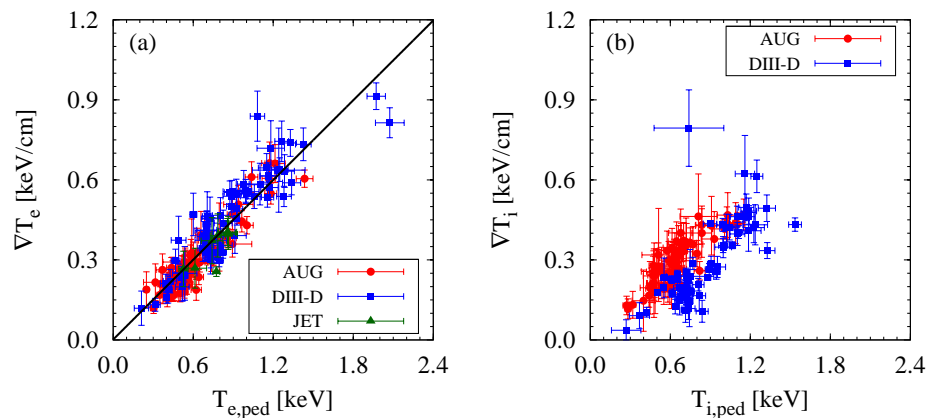

FIG. 2: Mean real space gradient in the steep gradient zone of the pedestal at the outer midplane plotted against the pedestal top value of electron temperature (a) and ion temperature (b). The same data was also shown in Fig. 1, where the separation was made by machine and not the measured parameter.
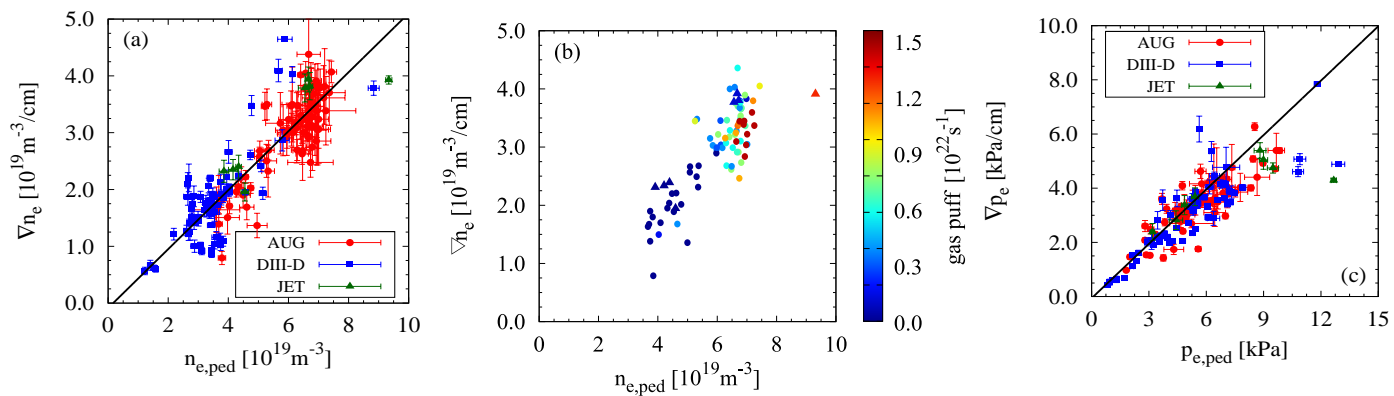

FIG. 3: Mean real space gradient in the steep gradient zone of the pedestal at the outer midplane plotted against the pedestal top value electron density (a) and electron pressure (c). Linear regressions to the data are indicated with solid black lines. (b) shows the same data as (a) for AUG (circles) and JET (triangles) with the gas puff as color code, the JET gas puff was scaled with 0.5 for a common color table. 

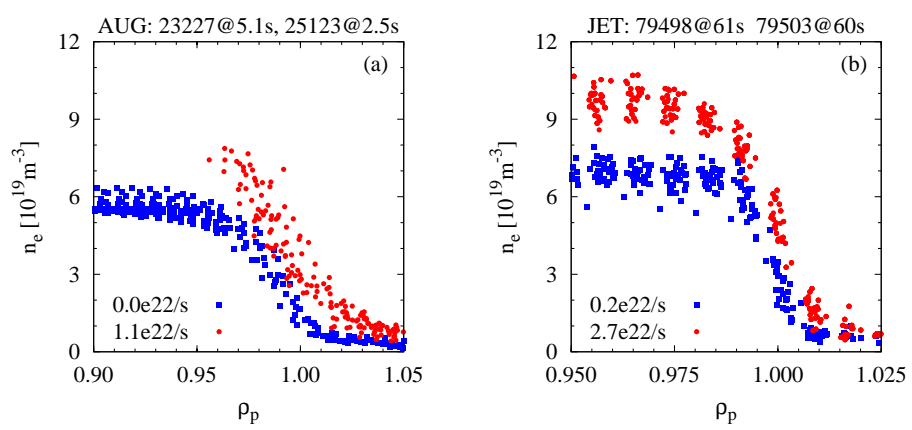

FIG. 4: Electron density profiles at the edge for AUG (Li-Beam) (a) and JET (Thomson scattering) (b) with low (blue) and high (red) gas fuelling. The profiles are taken in an interval of $150 \mathrm{~ms}(A U G)$ and $5 \mathrm{~s}$ (JET) in a stationary plasma - the instrument function of the JET TS was taken into account. The pedestal top for the high density case at AUG is defined by an edge interferometer channel which is not plotted. The JET profile is plotted on a different range of the normalized radius to illustrate the comparable real space gradients.

example for individual profiles is shown in Fig. 4. After aligning the profiles to the separatrix position determined by the temperature a similar result is obtained for AUG and JET. The separatrix position is defined as $T_{\mathrm{e}}=100 \pm 20 \mathrm{eV}$ for AUG and $T_{\mathrm{e}}=135 \pm 30 \mathrm{eV}$ for JET based on divertor measurements and transport parallel to the field lines [19]. The gas puff causes an increase of the separatrix density and the whole profile is shifted to higher densities with roughly constant width, where the width is defined from the pedestal top up to the separatrix. For AUG the increase in density at the separatrix and in the SOL is even more pronounced than at JET. This also indicates the different absolute impact of the gas puffing and confirms that external gas puffing cannot be used as comparable quantity in inter machine comparisons. The gas puffing at DIII-D is considerably lower - typically $20-30 \%$ of a gas puff in AUG - and does not show an influence on the pedestal top density in the presented data set. The alignment of the profiles was crosschecked with measurements of the divertor radiation which suggest higher neutral density in the divertor for both high fuelling cases compared to the low fuelling cases. This means a higher separatrix density is expected with increased fuelling and is consistent with the separatrix position obtained from the temperature profiles. The behaviour of constant density gradients in the pedestal and increased separatrix density was also observed in Alcator C-Mod [20]. In contrast to the observation at AUG and JET an increased pedestal top density could only be achieved for low plasma currents while for $I_{\mathrm{p}}>0.8 \mathrm{MA}$ the additional fuelling had little effect.

The gradient of the pedestal pressure in Fig. 3 (c) also shows a strong cor- 
relation with the pedestal top value. For all three machines the measurements are distributed around a gradient-top ratio which would correspond to a pedestal width of $\sim 1.5 \mathrm{~cm}$ and does show little systematic deviations. In particular, no influence of the machine size is observed in the JET data (green triangles). This is in contrast to measurements from Alcator C-Mod which typically show pedestal widths down to $0.5 \mathrm{~cm}$ [7]. Besides the smaller machine size, Alcator C-Mod differs from AUG, DIII-D and JET via higher magnetic field and significantly larger densities. When one excludes the results from the JET Thomson scattering with improved optics [21] the different machine size could nicely explain the difference in width. When considering also JET data the difference in density might also help to explain the deviation. The neutral penetration model [22] would go in the right direction and showed promising results [10], but was never tested on all 4 machines.

In this context it is important to note that the argumentation for the electron pressure width does not apply to $T_{\mathrm{e}}, n_{\mathrm{e}}$ or $T_{\mathrm{i}}$. In particular the ion temperature in Fig. 2 (c) shows a large variation in $T_{\mathrm{i}, \text { ped }} / \nabla T_{\mathrm{i}}$ which would suggest a larger ion temperature pedestal width for lower collisionality. This would be consistent with observations from JT60-U which showed a ion temperature pedestal width of about $4 \mathrm{~cm}$ [8] for plasmas with low collisionality. For the electron temperature $T_{\text {e,ped }} / \nabla T_{\mathrm{e}} \sim 2.0 \mathrm{~cm}$ was found, however this does not correspond to a pedestal width $\Delta$ as defined e.g. in [12]. The width is defined from the pedestal top up to the separatrix. Therefore, $T_{\mathrm{e}, \mathrm{ped}} / \nabla T_{\mathrm{e}}$ and $\Delta_{T \mathrm{e}}$ can differ from $0.1 \mathrm{up}$ to $1.0 \mathrm{~cm}$ depending on the gradient in the pedestal, as for small gradients the extend of $T_{\mathrm{e}, \mathrm{ped}} / \nabla T_{\mathrm{e}}$ into the scrape of layer becomes larger. For similar reasons $T_{\mathrm{e}, \text { ped }} / \nabla T_{\mathrm{e}} \neq T_{\mathrm{e}} / \nabla T_{\mathrm{e}}=L_{T \mathrm{e}} \neq \Delta_{T \mathrm{e}}$. For $n_{\mathrm{e}}$ with a finite separatrix density the same applies. However, the separatrix value of $p_{\mathrm{e}, \mathrm{sep}}=n_{\mathrm{e}, \mathrm{sep}} T_{\mathrm{e}, \mathrm{sep}}$ is quadratically reduced compared to the pedestal top, resulting in a necessary correction due to the separatrix of the order of only $1 \mathrm{~mm}$.

\section{Normalized pressure gradient}

For edge stability analysis a different representation of the gradients is of interest. This is the normalized total pressure gradient $\alpha$ which is used with the definition of Ref. [23] Eq. (42). $\alpha$ arises from the energy balance between destabilising energy available due to the gradient of the total pressure $p=p_{\mathrm{e}}+p_{\mathrm{i}}$ and the energy required for field line bending.

The ELM instability is currently best described with the peeling-ballooning theory [24] where a critical value of the edge current density $j$ and the normalized pressure gradient cause a combined peeling-ballooning mode to become unstable. For a given plasma discharge a stability boundary can be illustrated in a $j$ - $\alpha$ diagram. For most published cases of type-I ELMy H-modes the experimental point lies 

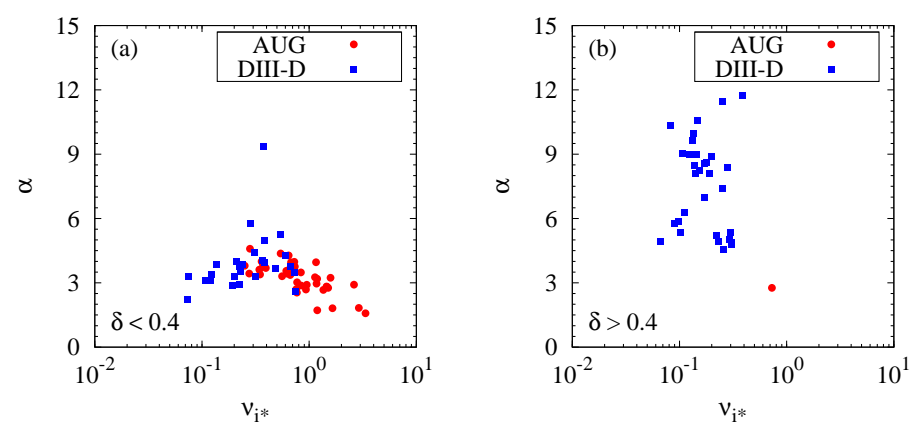

FIG. 5: Normalized pressure gradient $\alpha$ plotted against the collisionality for $\delta<0.4$ (a) and $\delta>0.4(b)$.

near the peeling-ballooning stability boundary [9, 25, 28, The position of the boundary in a $j$ - $\alpha$ diagram varies with plasma parameters as does the location of the operational point on this boundary. Often discussed quantities are the plasma collisionality, the plasma shape and the magnetic shear at the edge. The information obtained with the AUG, DIII-D database about the correlations of $\alpha$ with these parameters is documented in the remainder of this section.

Because of the differences in the ion and electron temperatures the approximation $p=2 p_{\mathrm{e}}$ is not used. To account for the differences in the temperature gradients the following approximation is applied

$$
\frac{\partial p}{\partial \Psi} \sim\left(1+\frac{1}{2} \frac{\frac{\partial T_{\mathrm{i}}}{\partial \Psi}}{\frac{\partial T_{\mathrm{e}}}{\partial \Psi}}+\frac{1}{2} \frac{T_{\mathrm{i}, \mathrm{ped}}}{T_{\mathrm{e}, \mathrm{ped}}}\right) \frac{\partial p_{\mathrm{e}}}{\partial \Psi} .
$$

This approximation implies a constant pedestal width of $T_{\mathrm{e}}$ and $n_{\mathrm{e}}$ in real space coordinates, considering Fig. 2 (a) and Fig. 3 (a) this is a reasonable assumption. Further, an effective charge number $Z_{\text {eff }}=1$ is used. In principle, a $Z_{\text {eff }}$ profile to determine $n_{\mathrm{i}}$ would be preferable. However, due to the large uncertainties of $Z_{\text {eff }}$, particularly at the edge, its inclusion would not improve the approximation for $\nabla p$. To quantify the possible error due to this approximation $Z_{\text {eff }}$ was varied by a factor of 4 and the change of $\alpha$ was calculated. In spite of the large variation in $Z_{\text {eff }} \alpha$ changed by less than $30 \%$. When comparing quantities which are similarly affected by $Z_{\text {eff }}$ the deviation from the correct values becomes even less.

The plasma shape divides the data set in two different regions, one of weak shaping and another of strong shaping. In Fig. 5 the collisionality dependence of $\alpha$ is shown for different triangularity. For low triangularity (a) only $\alpha$ values below 6 are reached. There is one exception which reaches $\alpha>9$, however, this is the only discharge in the set with a relatively large elongation of $\kappa>1.85$ and therefore also 

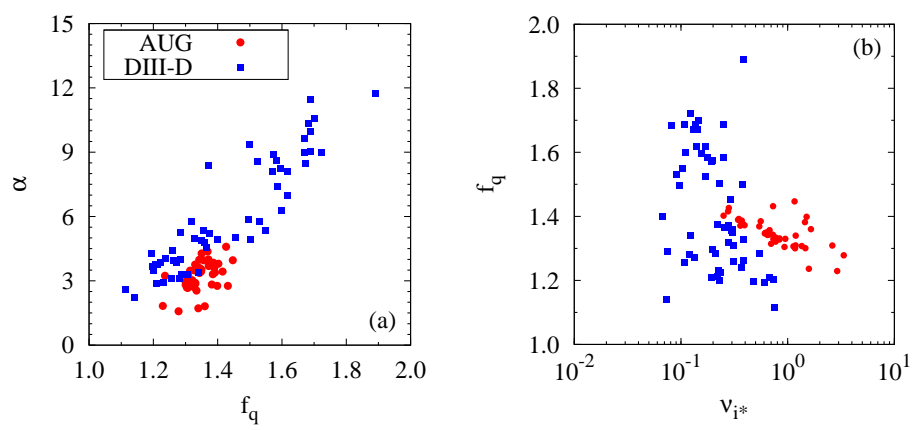

FIG. 6: Normalized pressure gradient $\alpha$ plotted against $f_{q}(a)$ and $f_{q}$ plotted against collisionality (b).

strongly shaped compared to a circular cross section. For high triangularity (b) larger values of $\alpha$ are possible, at the same time no correlation with collisionality is observed.

Another parameter which correlates with the normalized pressure gradient is $f_{\mathrm{q}}=$ $q_{95} / q_{\text {cyl }}$. With increasing $f_{\mathrm{q}}$ also larger values of $\alpha$ are possible as shown in Fig. 6] (a). Two regions can be identified, one where $f_{\mathrm{q}}$ increases but $\alpha$ varies little $\left(f_{\mathrm{q}}<1.5\right)$ and another were $\alpha$ strongly increases with $f_{\mathrm{q}}\left(f_{\mathrm{q}}>1.5\right)$. These regions also coincide with the regions of different shape in Fig. 5, In Fig. 6] (b) $f_{\mathrm{q}}$ is compared to the collisionality and both parameters do not show a notable correlation. Rather, a wide range of collisionalities can be covered while keeping $f_{\mathrm{q}}$ constant. The physical meaning of $f_{\mathrm{q}}$ can be understood when considering that the measurements of magnetic pick-up coils hold information about the current distribution in the plasma as long as the plasma is not circular but shaped [29]. All measurements presented here are based on non circular divertor plasmas with an x-point. Therefore, $q_{95}$ as reconstructed by equilibrium codes is closely connected to the plasma current within $95 \%$ of the normalized poloidal flux; while $q_{\text {cyl }}$ is proportional to the inverse of the total plasma current. Their ratio $f_{\mathrm{q}}$ is then a proxy for the amount of current situated within the plasma edge. Consequently $f_{\mathrm{q}}$ would hold information about the edge current density and the real area of the last $5 \%$ of the plasma radius. This area is essentially information about the plasma shape since the flux surfaces become more and more elliptical when approaching the plasma center. In this case when $f_{\mathrm{q}}$ represents a combination of edge current density and plasma shape Fig. 6 (a) would suggest also a strong link of the normalized pressure gradient and the edge current density, which can change the local shear significantly.

To assess the influence of the collisionality on the edge current density and understand Fig. 6 (b) it is useful to study the bootstrap current. With larger col- 
lisionality the bootstrap drive coefficients decrease [30]. However, this does not necessarily mean smaller bootstrap contribution to the edge current density. In contrary, $j_{\text {boot }}$ can even increase slightly. Following the ideas in [30], a simple estimate can be made. Assuming constant pressure and proportionality between gradients and pedestal height, a change of the collisionality from 0.5 to 1.7 will influence the bootstrap current by less than $5 \%$. This is because of the stronger boostrap drive due to the density compared to the one due to the temperature which compensates the overall reduction in the drive coefficients. Therefore, no distinct conclusion may be drawn from changes in collisionality to changes in bootstrap current. It depends strongly on how the variation in collisionality is achieved. For example, varying $\nu_{\star}$ with strong gas puffing will break the assumption of proportionality between gradients and pedestal height as discussed in Section 3 and most likely change the bootstrap current.

\section{Conclusion}

Surprisingly, the real space electron temperature gradient shows a linear dependence on $T_{\mathrm{e}, \mathrm{ped}}$, thus $T_{\mathrm{e}, \mathrm{ped}} / \nabla T_{\mathrm{e}} \sim$ const. The dependence of these two parameters is not disrupted by changes in plasma current, magnetic field, plasma shape or collisionality. The ion temperature gradient does not show such a linear dependence with the pedestal top value but exhibits significant deviations between machines which might be connected to different collisionality in the investigated plasmas. Changes in gas puffing have no impact on the electron density gradient, while the density pedestal top can be influenced by the gas-puff in a certain fuelling rate interval which differs for the machines. Also the electron pressure gradient appears to be independent of collisionality and plasma shape.

The normalized pressure gradient $\alpha$, which contains information of electrons and ions, does not exhibit a clear dependence on a single parameter, as was the case for the electron channel alone. A clear separation of high and low $\alpha$ is possible with the plasma shape, where high $\alpha$ are only achieved with strong shaping. There are indications that large elongation also opens up the access to high $\alpha$ as it is observed for the triangularity in the presented data set and previous studies [31]. Both can be expected from the peeling-ballooning theory [32, 33].

The different observations favour various mechanisms. The pedestal top values of electron and ion temperature and density can be individually transport limited, but together they still reach the peeling-ballooning stability limit.

The authors want to thank P.J. McCarthy for valuable discussions on the plasma equilibrium and also H. Zohm, K. Lackner and P.B. Snyder for their valuable input. This work was supported in part by EURATOM and carried out within the framework of the European Fusion Development Agreement. The views and opinions expressed herein do not 
necessarily reflect those of the European Commission. This work was supported in part by the U.S. Department of Energy under DE-FC02-04ER54698 and DE-FG02-95ER54309.

\section{References}

[1] SUTtRop, W. et al., Plasma Physics and Controlled Fusion 39 (1997) 2051.

[2] GROEBnER, R. J. et al., Plasma Physics and Controlled Fusion 48 (2006) A109.

[3] MAGGI, C. F., Nuclear Fusion 47 (2007) 535.

[4] GROEBNER, R. J. et al., Nuclear Fusion 50 (2010) 064002.

[5] BURCKHART, A. et al., Plasma Physics and Controlled Fusion 52 (2010) 105010.

[6] OSBORNE, T. H. et al., Fusion Energy 2002 (Proc. 19th Int. Conf. Lyon, 2002) (Vienna: IAEA) CD-ROM CN-94/CT-3 and http://www.iaea.org/programmes/ripc/physics/fec2002/html/fec2002.htm

[7] HUGHeS, J. W. et al., Physics of Plasmas 9 (2002) 3019.

[8] URANO, H. et al., Nuclear Fusion 48 (2008) 045008.

[9] MAGGI, C. et al., Nuclear Fusion 50 (2010) 025023.

[10] BEURSKENS, M. N. A. et al., Physics of Plasmas 18 (2011) 056120.

[11] WALK, J. R. et al., Nuclear Fusion 52 (2012) 63011.

[12] SCHNEIDER, P. A. et al., Plasma Physics and Controlled Fusion 54 (2012) 105009.

[13] KALLENBACH, A. et al., Journal of Nuclear Materials 337-339 (2005) 381.

[14] SNYDER, P. B. et al., Physics of Plasmas 16 (2009) 056118.

[15] GROEBNER, R. J. et al., Nuclear Fusion 49 (2009) 085037.

[16] NEU, R. et al., Journal of Nuclear Materials (2013) .

[17] VIEZZER, E. et al., Review of Scientific Instruments 83 (2012) 103501.

[18] GRIERSON, B. A. et al., Review of Scientific Instruments 81 (2010) 10 D735.

[19] KALLENBACH, A. et al., Nuclear Fusion 42 (2002) 1184.

[20] HUGHES, J. W. et al., Nuclear Fusion 47 (2007) 1057.

[21] FRASSINETTI, L. et al., Review of Scientific Instruments 83 (2012) 13506.

[22] MAHDAVI, M. A. et al., Physics of Plasmas 10 (2003) 3984.

[23] MILLER, R. L. et al., Physics of Plasmas 5 (1998) 973.

[24] CONNOR, J. W. et al., Physics of Plasmas 5 (1998) 2687.

[25] MAINGI, R. et al., Physical Review Letters 103 (2009) 18.

[26] SNYDER, P. et al., Nuclear Fusion 44 (2004) 320.

[27] SAARELMA, S. et al., Plasma Physics and Controlled Fusion 51 (2009) 035001.

[28] FENSTERMACHER, M. et al., Nuclear Fusion 45 (2005) 1493.

[29] MCCARTHY, P. J., Plasma Physics and Controlled Fusion 54 (2012) 015010.

[30] DUNNE, M. G. et al., Nuclear Fusion 52 (2012) 123014.

[31] OSBORNE, T. H. et al., Plasma Physics and Controlled Fusion 42 (2000) A175. 
[32] SNYDER, P. B. et al., Physics of Plasmas 9 (2002) 2037.

[33] AIBA, N. et al., Nuclear Fusion 52 (2012) 114002. 